\title{
Gutes Zeugnis für terminale palliative Sedierung
}

\author{
Einigen Krebspatienten mit unerträglichen Symptomen kann am Ende nur noch eine palliative \\ Sedierung helfen. Die Option ist sinnvoll - der Arzt muss aber unbedingt ordentlich dokumentieren.
}

\begin{abstract}
Bei manchen palliativmedizinisch betreuten Krebspatienten gelingt es in den Wochen vor dem Tod nicht mehr, Symptome wie Atemnot, Panik, Erstickungsangst oder Schmerz effektiv zu kontrollieren. Dann kann eine therapeutische palliative Sedierung hilfreich sein. Die Bewusstlosigkeit soll so leicht wie möglich, aber so tief wie für die Symptomkontrolle nötig sein.

Wie dies die Überlebenszeit der Patienten beeinflusst, untersuchten nun Palliativmediziner aus Japan. Zwischen 2012 und 2014 wurden an 58 stationären und ambulanten palliativmedizinischen Einrichtungen 1.827 Patienten im Alter von $\geq 20$ Jahren mit fortgeschrittenem Tumorleiden rekrutiert. Bei 269 von ihnen (15\%) wurde eine palliative terminale Sedierung durchgeführt. Diese Patienten verstarben im Mittel nach 22 Tagen, während Patienten ohne Sedie-
\end{abstract}

rung im Mittel noch 26 Tage lang lebten. Der Log-rank-Test ergab keine statistische Signifikanz $(\mathrm{p}=0,91)$. Für die Autoren stellt die terminale Sedierung durch spezialisierte Pallativteams deshalb eine sinnvolle Therapieoption in der Palliative Care dar.

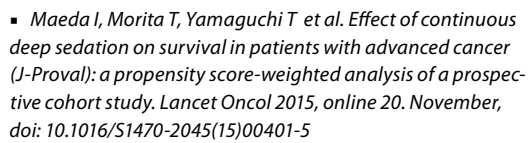
deep sedation on survival in patients with advanced cancer (J-Proval): a propensity score-weighted analysis of a prospective cohort study. Lancet Oncol 2015, online 20. November, doi: 10.1016/S1470-2045(15)00401-5

\section{KOMMENTAR}

Es stellt sich die Frage: Müssen sich Ärzte, die palliativ sedieren, den Vorwurf aktiver Sterbehilfe gefallen lassen? Nein, sicherlich nicht! Und zwar dann nicht, wenn das Behandlungsziel nicht "Verkürzung des Lebens", sondern "Symptomkontrolle und Verringerung von Leiden" ist, und wenn das Vorgehen mit dem Wunsch des Patienten bzw. seiner Patientenverfügung oder dem Votum seines Vor- sorgebevollmächtigten oder Betreuers übereinstimmt und alle anderen Behandlungsoptionen ausgeschöpft sind. In dieser Hinsicht bestärkt uns die japanische Studie.

Um das Vorgehen eindeutig gegenüber einer aktiven Sterbehilfe abzugrenzen, sind darüber hinaus empfehlenswert:

1. Dokumentation darüber, dass das Behandlungsziel nicht eine Lebensverkürzung, sondern eine Symptomlinderung ist, und dass diese mit anderen Behandlungsoptionen nicht zu erreichen war.

2. Dokumentation darüber, dass die palliative Sedierung dem (mutmaßlichen) Willen des Patienten entspricht.

3. Dokumentation darüber, dass mit der geringsten effektiven Sedativa-Dosierung gearbeitet wurde.

So eingesetzt ist die palliative Sedierung eine wertvolle Option, um ansonsten unerträgliches Leiden zu lindern.

Dr. med.J.Zeeh

\section{Steinbildung mal nicht in der Gallenblase}

Eine 88-jährige Frau wurde mit Erbrechen, Bauchschmerzen und geblähtem Abdomen in die Nothilfe gebracht. Sie berichtete, dass die Sympto-

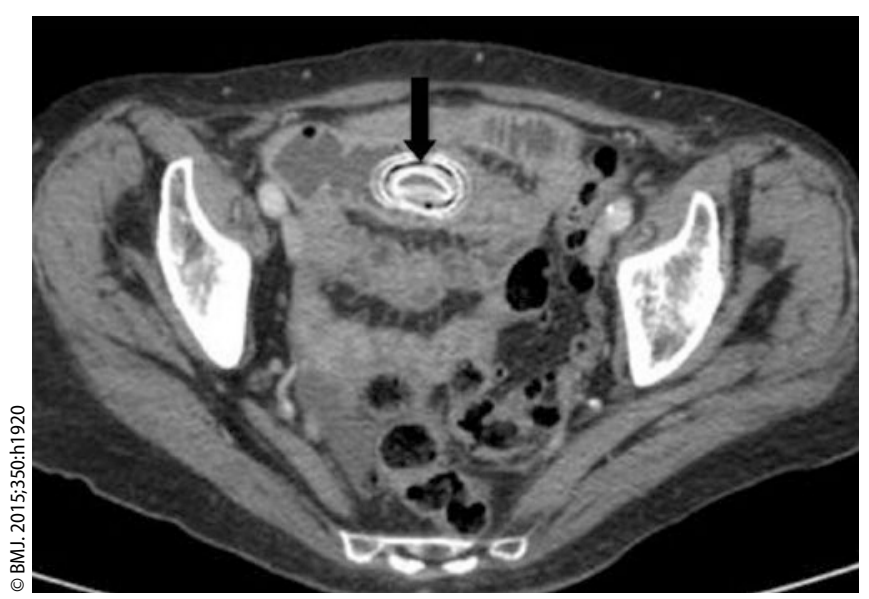

Obstruktion des distalen Dünndarms durch ein lamelliertes Konkrement im CT. matik bereits seit einer Woche bestand. Das Computertomogramm (CT) des Abdomens zeigte eine Obstruktion des distalen Dünndarms durch ein lamelliertes Konkrement (Abb. A, Pfeil). Das Bild passte zu einem Gallensteinileus, und daran dachte man auch als Erstes. Diese Verdachtsdiagnose wurde aber verworfen, weil zum einen keine Pneumobilie vorlag, und zum anderen auch Gallenblasensteine gefunden wurden, die eine andere Materialstruktur aufwiesen als das obstruierende Konkrement. Auf anderen Schnittebenen waren hingegen Konkremente mit ähnlicher Lamellierung darstellbar. Diese befanden sich allerdings in mehreren großen Jejunaldivertikeln.

Der große Enterolith wurde laparoskopisch aus dem distalen lleum entfernt. Die Patientin erholte sich in der Folge vollständig. Angesichts des reichlichen Steinvorrats in den Jejunaldivertikeln erschien es durchaus möglich, dass sich ein ähnliches Ereignis wiederholt. Aufgrund des fortgeschrittenen Lebensalters ließ man die Divertikel - und die alte Dame aber in Ruhe.

Prof. Dr. med. H. S. FüeßI

- Chanchlani Net al. (elizabeth.carty@bartshealth.nhs.uk).Small bowel diverticulosis. BMJ. 2015;350:h1920 